\title{
New Phase Synthesis in Surface Layer in the Conditions of Ion Implantation
}

\author{
Asfandyar Khan ${ }^{1,2, a)}$ and Anna G. Knyazeva $2,3,4$, b) \\ ${ }^{1}$ Department of Mathematics, Abdul Wali Khan University, Mardan, KPK, 23200, Pakistan \\ ${ }^{2}$ National Research Tomsk Polytechnic University, Tomsk, 634050, Russia \\ ${ }^{3}$ Institute of Strength Physics and Materials Science SB RAS, Tomsk, 634055, Russia \\ ${ }^{4}$ National Research Tomsk State University, Tomsk, 634050, Russia \\ a) Corresponding author: asfandyarmath@awkum.edu.pk \\ b) anna@ispms.tsc.ru
}

\begin{abstract}
The simple models for the formation of a new phase in a surface layer during ion implantation are discussed. The model of the new phase formation in the same conditions and considering the finiteness of relaxation time is suggested. The model parameters were evaluated using literature data. The stresses and strains in diffusion zone were calculated. The authors demonstrated that the concentration distribution and values of stresses depend on the relation between time scales of various physical processes.
\end{abstract}

Keywords: ion implantation, surface layer modification, relaxation time, phases formation

\section{INTRODUCTION}

The doping of new materials and the modification of surface layer are accompanied by various physical phenomena, including diffusion, chemical reactions, phase transition and heat transfer by different mechanisms, deformation, damage accumulation, fracture etc. All these phenomena are characterized by a different time and space scale, and they may have influence on each other, which will lead to the occurrence of numerous cross effects. This will affect the technology process. Nowadays, the study of complex technology processes of material synthesis and modification without mathematical modeling is not feasible, especially when the experiment involves multiple factors. For example, the phase synthesis in the conditions of ion implantation has some peculiarities. The reaction initiation is possible at moderate temperatures, because molecules and atoms in surface layer are activated by an external action [1,2]. The surface layer modification is determined by diffusion and chemical reactions with irreversible kinetics. The purpose of our investigation is the step-by-step construction of a model intended for the synthesis of a new phase in surface layer under the action of a particle beam. The model is based on the generalization of thermal elastic diffusion theory. Here we study the regularities of the change of surface layer composition implementing a diffusion problem with a singular chemical reaction and a variable shape of the particle beam pulse. We have evaluated stresses and strains in the diffusion zone for various kinetic parameters. In contrast to [3], the diffusion flux has finite relaxation time that is a distinctive feature of irreversible conditions. The parameters of the model are calculated for intermetallic systems. Particular problems are solved analytically and numerically.

\section{SIMPLE PROBLEMS}

When particle beam acts on the surface of a material, no chemical reactions occur, and the rate of particles along the beam is uniform. So we can assume the problem to be one-dimensional 


$$
\frac{\partial y_{A}}{\partial t}=D_{A} \frac{\partial^{2} y_{A}}{\partial x^{2}}, x=0: \mathbf{J}_{A}=-D_{A} \frac{\partial y_{A}}{\partial x}=q_{m}, x \rightarrow \infty: \mathbf{J}_{A}=0, t=0: y_{A}=0,
$$

where $y_{A}$ is the mole concentration of diffusing particles, $t$ is time, $x$ is the spatial coordinate in the direction of a particle beam action, $D_{A}$ is diffusion coefficient, $q_{m}$ is the particle beam density. The problem has the solution that is described in literature

$$
y_{A}=2 \frac{q_{m}}{\sqrt{D_{A}}} \sqrt{\frac{t}{\pi}}\left[\exp \left(-\frac{x^{2}}{4 D_{A} t}\right)-\frac{x}{\sqrt{D_{A}}} \operatorname{erfc}\left(\frac{x}{2 \sqrt{D_{A} t}}\right)\right] .
$$

When the chemical reaction is possible in the surface layer, the particles are consumed to form a new phase.

Let us assume that the chemical reaction occurs according to the simplest scheme $A+B \rightarrow C$, where $B$ corresponds to the substance contained in an initial specimen and $C$ is the reaction product.

An additional component appears in the diffusion equation and we come to the second simple problem

$$
\frac{\partial y_{A}}{\partial t}=D_{A} \frac{\partial^{2} y_{A}}{\partial x^{2}}-k_{A} y_{A}, x=0: \mathbf{J}_{A}=-D_{A} \frac{\partial y_{A}}{\partial x}=q_{m}, x \rightarrow \infty: \mathbf{J}_{A}=0, t=0: y_{A}=0
$$

The exact solution of this problem has the following form:

$$
y_{A}=\frac{q_{m}}{2 \sqrt{D_{A} k_{A}}}\left[\exp \left(-x \sqrt{\frac{k_{A}}{D_{A}}}\right) \operatorname{erfc}\left(\frac{x}{2 \sqrt{D_{A} t}}-\sqrt{k_{A} t}\right)-\exp \left(x \sqrt{\frac{k_{A}}{D_{A}}}\right) \operatorname{erfc}\left(\frac{x}{2 \sqrt{D_{A} t}}+\sqrt{k_{A} t}\right)\right] .
$$

Note that the previous formula (2) represents the limit of this solution for $k_{A} \rightarrow 0$.

Because the implanted particles are consumed for a new phase formation, their concentration decreases in the volume. The concentration of products changes according to equation $\partial y_{C} / \partial t=\omega_{C}, \omega_{C}=k_{C} y_{A}$.

For the first order simple reaction we have evaluated the product concentration using the Laplace transform:

$$
\begin{aligned}
y_{C}= & \frac{q_{m}}{2 \sqrt{D_{A} k_{A}}} k_{C}\left[\exp \left(-x \sqrt{\frac{k_{A}}{D_{A}}}\right) \int_{0}^{t}(t-\tau) \operatorname{erfc}\left(\frac{x}{2 \sqrt{D_{A} \tau}}-\sqrt{k_{A} \tau}\right) \mathrm{d} \tau\right. \\
& \left.-\exp \left(x \sqrt{\frac{k_{A}}{D_{A}}}\right) \int_{0}^{t}(t-\tau) \operatorname{erfc}\left(\frac{x}{2 \sqrt{D_{A} \tau}}+\sqrt{k_{A} \tau}\right) \mathrm{d} \tau\right] .
\end{aligned}
$$

\section{DIFFUSION AND REACTION OF MASS FLUX DEPENDING ON TIME}

For the second problem, we assume that

$$
\mathbf{J}_{A}=-D_{A} \frac{\partial y_{A}}{\partial x}=q_{m} \exp (-A t)
$$

The solution of the new problem can be obtained by means of the Laplace transform:

$$
\begin{gathered}
Y_{\mathrm{A}}^{0}=\frac{q_{m}}{\sqrt{D_{A}}} \int_{0}^{t}\left\{\frac { A \operatorname { e x p } ( - A ( t - \tau ) ) - k _ { A } \operatorname { e x p } ( - k _ { A } ( t - \tau ) ) } { A - k } \left\{\frac{1}{\sqrt{\pi \tau}} \exp \left[-\left(\frac{x^{2}}{4 D_{A} \tau}+k_{A} \tau\right)\right]\right.\right. \\
\left.+\frac{\sqrt{k_{A}}}{2}\left[\exp \left(-x \sqrt{\frac{k_{A}}{D_{A}}}\right) \operatorname{erfc}\left(\frac{x}{2 \sqrt{D_{A} \tau}}-\sqrt{k_{A} \tau}\right)-\exp \left(x \sqrt{\frac{k_{A}}{D_{A}}}\right) \operatorname{erfc}\left(\frac{x}{2 \sqrt{D_{A} \tau}}+\sqrt{k_{A} \tau}\right)\right]\right\} \mathrm{d} \tau .
\end{gathered}
$$

In this case, the concentration distribution depends on the correlation between typical scales of various physical quantities (for example, diffusion rate, reaction rate and rate of particle implantation) that are affected by the parameter $A$. The dependence of ion source density on time may vary. For instance,

$$
\mathbf{J}_{A}=-D_{A} \frac{\partial y_{A}}{\partial x}=q_{m}(\alpha+\beta t) \text { or } \mathbf{J}_{A}=-D_{A} \frac{\partial y_{A}}{\partial x}=q_{m}(1-\cos (\gamma t)) \text { or } \mathbf{J}_{A}=-D_{A} \frac{\partial y_{A}}{\partial x}=\frac{q_{m}}{1+\delta t} .
$$

The analytical solution of the said problems is not feasible or too cumbersome, hence not functional; so this problem ought to be solved numerically. 


\section{GENERAL STATEMENT OF PROBLEM}

We have the body with the surface uniformly treated by a particle beam with a given intensity and time structure. In the treated zone a chemical reaction of the following type can occur: $v_{A} A+v_{B} B \rightarrow v_{C} C$, where $v_{A}, v_{B}, v_{C}$ are stoichiometric coefficients. The concentration of particle implanted into the surface layer can change due to diffusion and chemical reaction. The balance equation has the following form

$$
\frac{\partial y_{A}}{\partial t}=-\frac{\partial}{\partial x} \mathbf{J}_{A}+\omega_{A} \text {. }
$$

Since the specific time is very small, we use the generalized equation for diffusion flux

$$
\mathbf{J}_{A}=-D_{A} \frac{\partial y_{A}}{\partial x}-t_{A} \frac{\partial \mathbf{J}_{A}}{\partial t} .
$$

The boundary and initial conditions are as follows:

$$
x=0: \mathbf{J}_{A}=q_{m} f(t), x \rightarrow \infty: y_{A}=0 \text { or } \mathbf{J}_{A}=0, t=0: y_{A}=0, \frac{\partial \mathbf{J}_{A}}{\partial t}=0 .
$$

Here $y_{A}\left[\mathrm{~mol} / \mathrm{m}^{3}\right]$ is the concentration of substance $A, \mathbf{J}_{A}$ is the substance flux $\left[\mathrm{mol} /\left(\mathrm{m}^{3} \mathrm{~s}\right)\right] ; \omega_{A}=-k_{A} y_{A}=-\omega v_{A}$ $[1 / \mathrm{s}] ; k_{A}=v_{A} k$-rate constant for chemical reaction, $t_{A}$ is relaxation time and $D_{A}$ is diffusion coefficient $\left[\mathrm{m}^{2} / \mathrm{s}\right]$. The simplification of equation (7) and (6) yields

$$
\frac{\partial \mathbf{J}_{A}}{\partial z}=-D_{A} \frac{\partial^{2} y_{A}}{\partial z^{2}}+t_{A}\left(\frac{\partial^{2} y_{A}}{\partial t^{2}}-\frac{\partial \omega_{A}}{\partial t}\right) .
$$

Using the flux derivative from (6) we obtain

$$
t_{A}\left(\frac{\partial^{2} y_{A}}{\partial t^{2}}-\frac{\partial \omega_{A}}{\partial t}\right)+\frac{\partial y_{A}}{\partial t}=D_{A} \frac{\partial^{2} y_{A}}{\partial z^{2}}+\omega_{A} .
$$

According to the mass action law, the chemical reaction rate can be written as $\omega=k y_{A}{ }^{v_{A}} y_{B}{ }^{v_{B}}$, where $y_{B}$ is the concentration of basic substance, but in the majority of cases we can assume $\omega=k^{\prime} y_{A}$.

To complete the problem formulation, we substitute the kinetic equation for the reaction product to the problem (9) and (8): $\partial y_{C} / \partial t=\omega_{C}$, where $\omega_{C}=k_{C} \omega=v_{C} k \omega$. We have the mixture of three components $A+B+C$. When the species $B$ is mobile we can assume that the relaxation time for this element $t_{B}$ differs from $t_{A}$. Hence, we obtain the second equation for diffusion flux and diffusion equation:

$$
\mathbf{J}_{B}=-D_{B} \frac{\partial y_{B}}{\partial z}-t_{B} \frac{\partial \mathbf{J}_{B}}{\partial t} \text { and } t_{B}\left(\frac{\partial^{2} y_{B}}{\partial t^{2}}-\frac{\partial \omega_{B}}{\partial t}\right)+\frac{\partial y_{B}}{\partial t}=D_{B} \frac{\partial^{2} y_{B}}{\partial x^{2}}+\omega_{B} \text {, respectively }
$$

For example, for the systems $\mathrm{Ti}-\mathrm{Ni}, \mathrm{Ti}-\mathrm{Al}, \mathrm{Ni}-\mathrm{Al}$ we can suggest the simplified reaction schemes: $\mathrm{Ti}+\mathrm{Ni} \rightarrow \mathrm{TiNi}, \mathrm{Ti}+\mathrm{Al} \rightarrow \mathrm{TiAl}, \mathrm{Ni}+\mathrm{Al} \rightarrow \mathrm{NiAl}$, respectively. In this case, the absolute values of stoichiometric coefficients equal to unity $v_{A}=v_{B}=v_{C}=1$, and the equation for reaction product for all systems will be the following:

$$
\omega=k y_{A} y_{B}, \text { and } \frac{\partial y_{C}}{\partial t}=k y_{A} y_{B} .
$$

The diffusion equations are as follows:

$$
\begin{gathered}
t_{A} \frac{\partial^{2} y_{A}}{\partial t^{2}}+\left(1+t_{A} k y_{B}\right) \frac{\partial y_{A}}{\partial t}=D_{A} \frac{\partial^{2} y_{A}}{\partial x^{2}}-k y_{A}\left[y_{B}+t_{A} \frac{\partial y_{B}}{\partial t}\right], \\
t_{B} \frac{\partial^{2} y_{B}}{\partial t^{2}}+\left(1+t_{B} k y_{A}\right) \frac{\partial y_{B}}{\partial t}=D_{B} \frac{\partial^{2} y_{B}}{\partial x^{2}}-k y_{B}\left[y_{A}+t_{B} \frac{\partial y_{A}}{\partial t}\right] .
\end{gathered}
$$

Assuming $f(t)=\exp (-A t)$, the equation (8) gives

$$
q_{m}\left(1-t_{A} A\right) \exp (-A t)=-D_{A} \frac{\partial y_{A}}{\partial x}, x=0
$$

At this point $\mathbf{J}_{B}=0$. The last boundary condition and the initial condition are: 


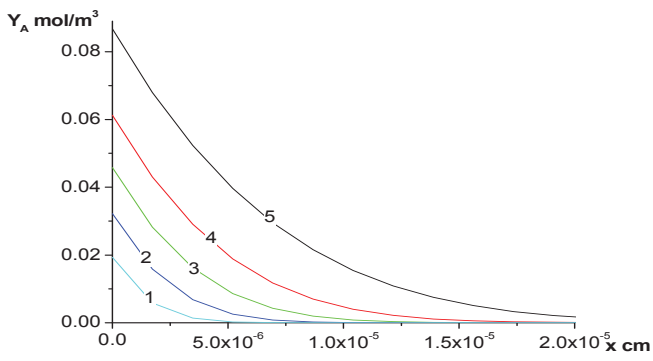

(a)

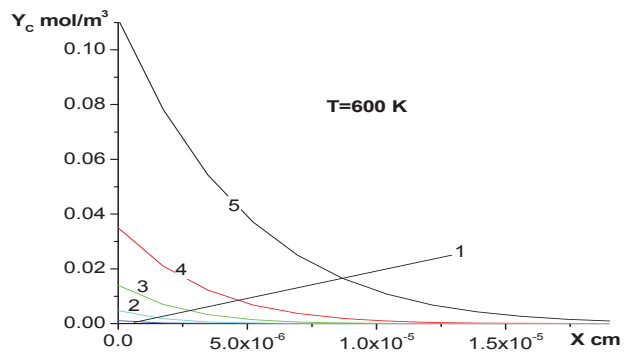

(b)

FIGURE 1. Concentration distribution in specimen at different moment of time: (a) implanted element, (b) reaction product.

$$
\begin{aligned}
& t_{A}=t_{B}=0, T=600 \mathrm{~K}, A=10 . t=5.99 \cdot 10^{-7}(1), 1.49 \cdot 10^{-6}(2), 2.99 \cdot 10^{-6}(3), 5.50 \cdot 10^{-5}(4), 1.20 \cdot 10^{-5} \mathrm{~s}(5) \\
& x \rightarrow \infty: y_{A}=0, y_{B}=0 \text { or } \mathbf{J}_{A}=0, \mathbf{J}_{B}=0, t=0: y_{A}=0, \frac{\partial \mathbf{J}_{A}}{\partial t}=0, \quad y_{B}=y_{B 0}, \frac{\partial \mathbf{J}_{B}}{\partial t}=0, y_{C}=0 .
\end{aligned}
$$

The problem (10)-(12) with boundary and initial conditions can be solved numerically.

In this model, we have different relaxation times $t_{A}, t_{B}$, impulse duration $t_{i}$, chemical reaction time $t_{\mathrm{ch}}$ and specific diffusion time $t_{D}$ for substances $A$ and $B$. The correlation between these times will determine the concentration distribution. Having that $t_{\mathrm{ch}}=y_{B 0} / k$ and assuming $k=k_{0} \exp \left(-E_{R} /(R T)\right), k_{0} / y_{B 0}=2 \cdot 10^{16} 1 / \mathrm{s}$ and $E_{R}=1.181 \cdot 10^{5} \mathrm{~J} / \mathrm{mol}$ [4]. We find $t_{\mathrm{ch}}=9.7 \cdot 10^{-7} \mathrm{~s}$ at the temperature $T=600 \mathrm{~K}$. Diffusion coefficients depend on temperature in accordance with Arrhenius law, i.e. $D_{k}=D_{k 0} \exp \left(-E_{D} /(R T)\right), k=A, B$. For nickel and titanium [5] $D_{\mathrm{Ni}}=1.9 \exp (-279700 /(R T))$ and $D_{\mathrm{Ti}}=1.09 \exp (-251200 /(R T))+3.58 \cdot 10^{-4} \exp (-130600 /(R T)) \mathrm{cm}^{2} / \mathrm{s}$. At the temperature of $T=600 \mathrm{~K}$ we get $D_{A}=1.51 \cdot 10^{-15} \mathrm{~cm}^{2} / \mathrm{s}$ and $D_{B}=8.2 \cdot 10^{-25} \mathrm{~cm}^{2} / \mathrm{s}$.

Here we assume that the activation energy reduction takes place four times. For $T=600 \mathrm{~K}$ the values of diffusion coefficients are close to those of previous simple problems: $D_{A}=4.21 \cdot 10^{-6} \mathrm{~cm}^{2} / \mathrm{s}$ and $D_{B}=1.54 \cdot 10^{-6} \mathrm{~cm}^{2} / \mathrm{s}$.

\section{ANALYSIS OF RESULTS}

The concentration distributions for $t_{A}=t_{B}=0$ are presented in Fig. $1\left(q_{m}=2.5 \cdot 10^{-4} \mathrm{~mol} /\left(\mathrm{m}^{2} \mathrm{~s}\right) ; T=600 \mathrm{~K}\right.$ and $A=10)$. In this case $t_{\mathrm{ch}}=9.7 \cdot 10^{-7} \mathrm{~s}$.

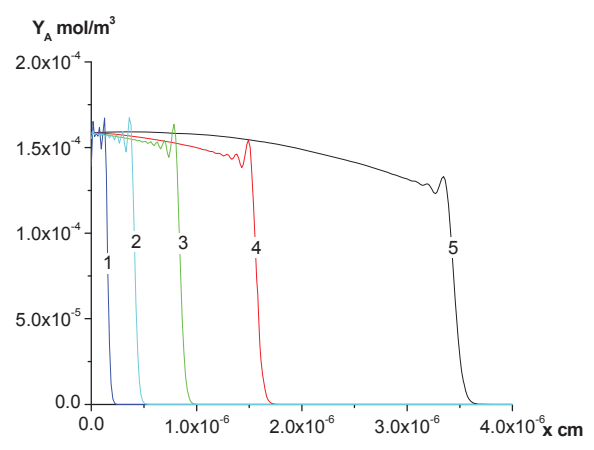

(a)

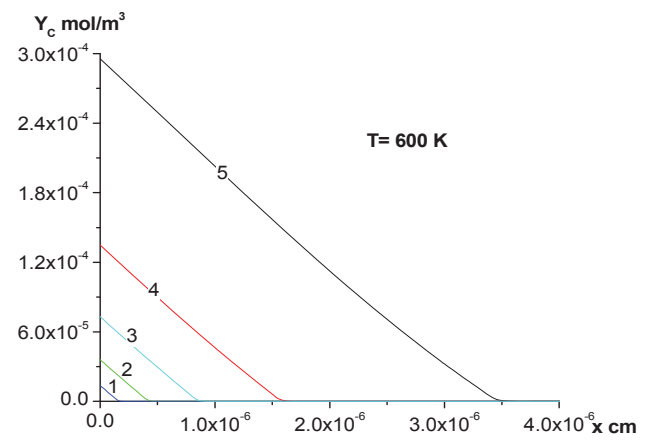

(b)

FIGURE 2. Concentration distribution in specimen at different moment of time. a) implanted element, b) reaction product. $t_{A}=5 \cdot 10^{-5}, t_{B}=10^{-5} \mathrm{~s} ; T=600 \mathrm{~K}, A=10 . t=5.99 \cdot 10^{-7}(1), 1.49 \cdot 10^{-6}(2), 2.99 \cdot 10^{-6}(3), 5.50 \cdot 10^{-5}(4), 1.20 \cdot 10^{-5} \mathrm{~s}(5)$ 


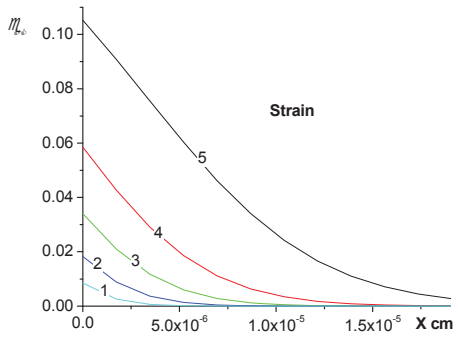

(a)

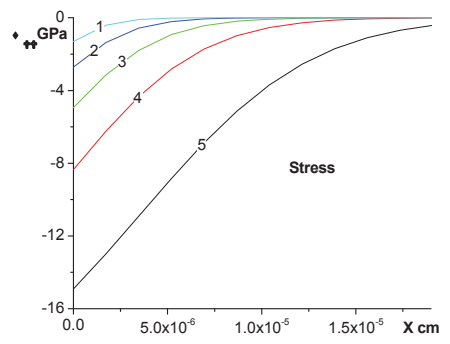

(b)

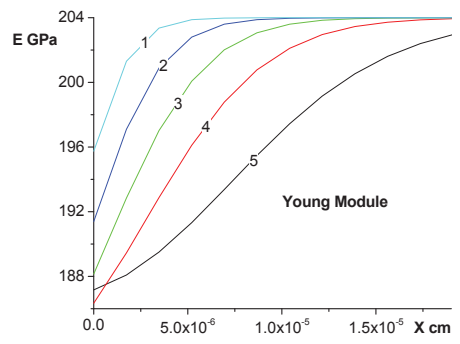

(c)

FIGURE 3. Strains, stresses and Young modulus in surface layer at different moment of time. $t_{A}=t_{B}=0, T=600 \mathrm{~K}, A=10 . t=$ $5.99 \cdot 10^{-7}(1), 1.49 \cdot 10^{-6}(2), 2.99 \cdot 10^{-6}(3), 5.50 \cdot 10^{-5}(4), 1.20 \cdot 10^{-5} \mathrm{~s}(5)$

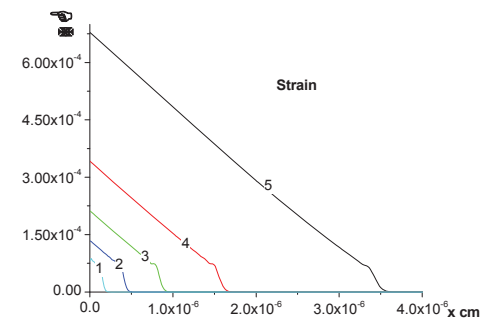

(a)

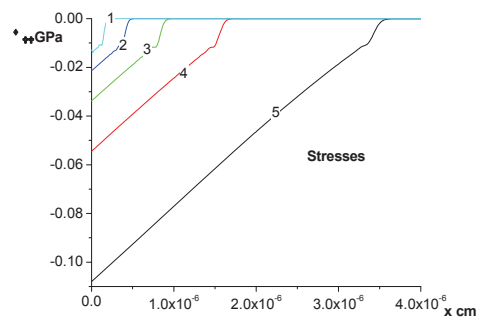

(b)

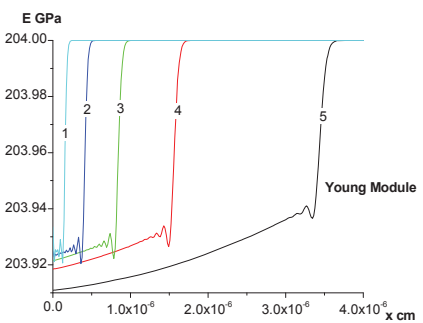

(c)

FIGURE 4. Strains, stresses and Young modulus in surface layer at different moment of time. $t_{A}=5 \cdot 10^{-5} ; t_{B}=10^{-5} \mathrm{sec} ; T=$ $600 \mathrm{~K}, A=10 . t=5.99 \cdot 10^{-7}(1), 1.49 \cdot 10^{-6}(2), 2.99 \cdot 10^{-6}(3), 5.50 \cdot 10^{-5}(4), 1.20 \cdot 10^{-5} \mathrm{~s}(5)$

Stresses in the reaction zone perpendicular to the particle beam reach large values. The Young modulus changes very quickly, because the properties of materials are close.

\section{CONCLUSION}

As a result, we have extended the existing model to a unique simple model describing the formation of a new phase at the initial stage of ion implantation. The known problem is a special case for our model. We have discussed two cases: a) when the time of relaxation is zero and b) when relaxation time is non-zero. The difference is clear from the figures of concentration and product distribution. Therefore, the finiteness of the relaxation time changes the concentration distribution in diffusion zone in comparison with common diffusion problems. The stresses, strains and Young modulus can reach large values. The model can be applied to various chemical systems.

\section{REFERENCES}

1. J. Jagielski, Technologue, 33, 509 (1999).

2. A. G. Knyazeva and S. G.Psakhie, J. Appl. Mech. Tech. Phys. 50(1), 118 (2009).

3. A. G. Knyazeva, Proc. Int. Conf. "Chemical and Radiation Physics”, Moscow, 2009, p. 125-129.

4. I. A. Kurzina, E. V. Kozlov, and Yu. P. Sharkeev, Gradient Surface Layers On The Base Intermetallic Particles. Synthesis, Structure, Properties (Izd-vo NTL, Tomsk, 2013).

5. A. D. Pogrebnjak and E. A. Bazyl, Sci. Dirt. 64(1), 1 (2001). 